\title{
Review and report of the role of Breast Milk Stem Cell in balancing immunomodulation after cardio pulmonary bypass
}

\begin{abstract}
Congenital Cardiac malformations and their repair are fraught with challenges. The complexity of care and issues thereof need multiple teams and coordinated care at the bedside. The frail infants and their well-being hangs by a very fragile balance in which breast milk and the stem cells therein have a more profound effect, heretofore unrecognized and less studied. Nestin positive stem cells in human breast milk and the phenomenon of microchimerism form foundational support for the immune effects after cardiopulmonary bypass for open heart surgery in those babies in whom early enteral nutrition with human breast milk is practiced in institutionalized manner as a care protocol. In a brief systematic review of literature (pubmed), we report the absence of any such study. The benefits of this culturally acceptable and low cost effective strategy goes a long way in improving outcomes in resource poor settings and high volume centres.
\end{abstract}

Volume 4 Issue 4 - 2018

\author{
Ramesh P Menon,' Gaurav Kaushik² \\ ${ }^{1}$ All India Institute of Medical Sciences, New Delhi, India \\ ${ }^{2}$ The University of Kansas Medical Centre, Department of \\ Surgery, USA
}

\begin{abstract}
Correspondence: Gaurav Kaushik, Senior Scientist, The University of Kansas Medical Centre, Department of Surgery, 390 I Rainbow Boulevard, Kansas City, KS, 66160, USA, Email gauravkaushik21@gmail.com

Ramesh P Menon (MD), Assistant Professor of Pediatrics, All India Institute of Medical Sciences, Ansari Nagar, New Delhi-I I0029, India, Email rpmpgi@gmail.com
\end{abstract}

\section{Mini review}

Young infants and newborn babies born with cardiac malformations have to undergo complex cardiac surgery with cardiopulmonary bypass in order to survive. The developmental stage of immune function in these cachectic infants and the cytokine and inflammatory storm that ensues after prolonged cardiopulmonary bypass, predisposes them to sepsis syndrome and multiorgan dysfunction in the immediate postoperative period as well as in the short term. ${ }^{1,2}$

In resource constrained and high volume settings, it literally translates to (ab) use of highly toxic antibiotics like colistin, highdose carbapenems and others (like capsofungin, ulinastatin etc). ${ }^{3}$ The postoperative morbidity and mortality due to infections become unacceptably high besides fostering multidrug resistance amongst bugs. In such circumstances, the role of enteral nutrition is being studied and has shown beneficial effects even in the critical care settings. ${ }^{4}$ The nutrition support has now become a key component in the postoperative care of these critically ill patients. This led us to review the metabolic and inflammatory burst induced by cardiac surgery. ${ }^{5}$ However, the role and function of human (breast) milk as a whole some stem cell source in infants after open heart surgery or after cardiopulmonary bypass has not been studied yet.

The advance in the research on uniqueness of the human milk for each mother-baby dyad has highlighted the cellular profile and immunological function of the tissue. Breast milk is now understood to be a "live" liquid tissue transfer. It's quality and quantity is modulated by various determinant factors including specific needs of the specific baby (viz. gestation, gender, infection status etc). For example, if the preterm baby is predisposed to infection, the immune profile of breast milk of the mother of the baby has more leucocytes/ lymphocytes. At other times, it has a different constitution including micro and macro nutrients.

The characterisation of stem cells in human milk has been a recent endeavour. ${ }^{6}$ They were initially reported by a team of scientists in 2007 as nestin positive stem cells in mother's milk of term infants. ${ }^{7}$
According to these investigators, they exhibit characteristics of pleuripotent stem cells (like hESC: hBSC) with no predetermined destination organogenesis. It is hypothesised that following ingestion, breast milk stem cells promote tissue homeostasis and regeneration anywhere in the infant's body, including the brain. ${ }^{8-11}$ Breastfeeding allows maternal leukocytes to habitate the infant's body in a process known as microchimerism. ${ }^{12}$ This mechanism prompted search for similar actions of breast milk stem cells. Mouse models demonstrated that the ingested breast milk stem cells survive the gastrointestinal tract and spread throughout the body where they are then found as differentiated cells within different organs of the mouse pup. ${ }^{13}$ It is now understood that breast milk ingestion in early infancy during periods of critical sickness can decrease disease later in life (clear mechanistic linkages do not exist). These mechanisms are explained through bioactive breast milk stem cells and components forming the metobolomics-microbiomics link in the native immune development of the newly born. These (linking ring) facilitate the replenishment and restoration of damaged tissues. ${ }^{14,15}$ These findings could be particularly important for cardiac infants, whose vulnerabilities make their needs particularly great.

Infants and neonates undergoing cardiac surgery experience a complex systemic inflammatory response syndrome ${ }^{5}$ (literally an inflammatory storm), which manifests as pyrexia, tachycardia, leukocytosis, hypotension, edema, and organ failure. It also tips the developing immature immune system into a Th2 response (antibody dominant) in the early postoperative phase. The surgical tissue handling, exposure to foreign surfaces, nonpulsatile circulation, ischemia -reperfusion injury and under nutrition/cachexia are all triggers for the inflammatory storm in the fragile young baby undergoing open heart surgery under cardiopulmonary bypass with aortic crossclamp.

In recent years, there has been interest in Early Enteral nutrition (using breast milk) soon after cardiac surgery in very young infants. ${ }^{16}$ The practise of prolonged fasting or total parenteral nutrition for the immediate postoperative period or till the sternum is closed is 
undergoing a change in practise to early introduction of expressed human milk (before surgery or soon after by the mother) and escalation of feed volume to meet caloric and metabolic requirements. This has resulted in no significant increase in postoperative complications (chylothorax, aspiration pneumonia, dyselectrolytemia) and even infection rates have reduced. The leukocyte count and period of ICU and hospital stay has significantly reduced. The duration of mechanical ventilation, length of ICU stay (LOIS), length of hospital stay (LOHS), infection rate, and mortality rate were lower in the intervention group (early breast milk feeding group) compared to the control group. The IL-10 (antiinflammatory) and other cytokine profile of the mother's milk provide a balancing immunomodulation for the baby. It has also been observed that (personal observation) that the monocyte counts and neutrophil percentages normalise in these infants soon after initiation of breast milk feeding in the post (cardiac) operative phase.

In a systematic review ${ }^{4}$ published this year, studies on protocols of hypocaloric feeding or amount of macronutrients in critical illness with special focus on adult patients after cardiac surgery, no sufficiently designed, adequately powered, randomized controlled trials investigating the effect of nutritional therapy in cardiac patients after surgery were available. In resource constrained settings with high volumes of this cohort of infants, a systematic enquiry into this domain of benefits of human milk feeding would be a paradigm shift, second to no other.

\section{Acknowledgements}

None.

\section{Conflicts of interest}

Author declares no conflicts of interest.

\section{References}

1. Justus G, Walker C, Rosenthal LM, et al. Immunodepression after CPB: Cytokine dynamics and clinics after pediatric cardiac surgery-A prospective trial. Cytokine. 2017;S1043-S4666(17):30085-30086.

2. Gaudriot B, Uhel F, Gregoire M, Gacouin A, Biedermann S, Roisne A, Flecher E, Le Tulzo Y, Tarte K, Tadié JM. Immune Dysfunction After Cardiac Surgery with Cardiopulmonary Bypass: Beneficial Effects of Maintaining Mechanical Ventilation. Shock. 2015;44(3):228-233.
3. Sahu MK, Siddharth B, Choudhury A, et al. Incidence, microbiological profile of nosocomial infections, and their antibiotic resistance patterns in a high volume Cardiac Surgical Intensive Care Unit. Annals of Cardiac Anaesthesia. 2016;19(2):281-287.

4. Introduction to Human Nutrition. 2nd ed. In: Michael J Gibney, Susan A Lanham-New, Aedin Cassidy, editors. A John Wiley \& Sons; 2009:1-386.

5. Hill A, Nesterova E, Lomivorotov V, et al. Current Evidence about Nutrition Support in Cardiac Surgery Patients-What Do We Know? Nutrients. 2018;10(5):E597.

6. Briere CE, Mc Grath JM, Jensen T, et al. Breast Milk Stem Cells: Current Science and Implications for Preterm Infants. Adv Neonatal Care. 2016;16(6):410-419.

7. Cregan MD, Fan Y, Appelbee A, et al. Identification of nestin-positive putative mammary stem cells in human breastmilk. Cell Tissue Res. 2007;329(1):129-136.

8. Fan Y, Chong YS, Choolani MA, et al. Unravelling the mystery of stem/ progenitor cells in human breast milk. PLoS One. 2010;5(12):e14421.

9. Reali A, Puddu M, Pintus MC, et al. Multipotent stem cells of mother's milk. J Pediatr Neonat Individual Med. 2016;5(1):e050103.

10. Hassiotou F, Beltran A, Chetwynd E, et al. Breast milk is a novel source of stem cells with multi-lineage differentiation potential. Stem Cells. 2012;30(10):2164-2174.

11. Twigger AJ, Hodgetts S, Filgueira L, et al. From breast milk to brains: the potential of stem cells in human milk. J Hum Lact. 2013;29(2):136-139.

12. Ichinohe T. Long-term feto-maternal microchimerism revisited: Microchimerism and tolerance in hematopoietic stem cell transplantation. Chimerism. 2010;1(1):39-43.

13. Cabinian A, Sinsimer D, Tang M, et al. Transfer of Maternal Immune Cells by Breastfeeding: Maternal Cytotoxic T Lymphocytes Present in Breast Milk Localize in the Peyer's Patches of the Nursed Infant. PLoS One. 2016;11(6):e0156762.

14. Bardanzellu F, Fanos V, Strigini FAL, et al. Human Breast Milk: Exploring the Linking Ring Among Emerging Components. Front Pediatr. 2018;6:215.

15. Witkowska-Zimny M, Kaminska-El-Hassan E. Cells of human breast milk. Cellular \& Molecular Biology Letters. 2017;22:11.

16. Sahu MK, Singal A, Menon R, et al. Early enteral nutrition therapy in congenital cardiac repair postoperatively: A randomized, controlled pilot study. Annals of Cardiac Anaesthesia. 2016;19(4):653-661. 